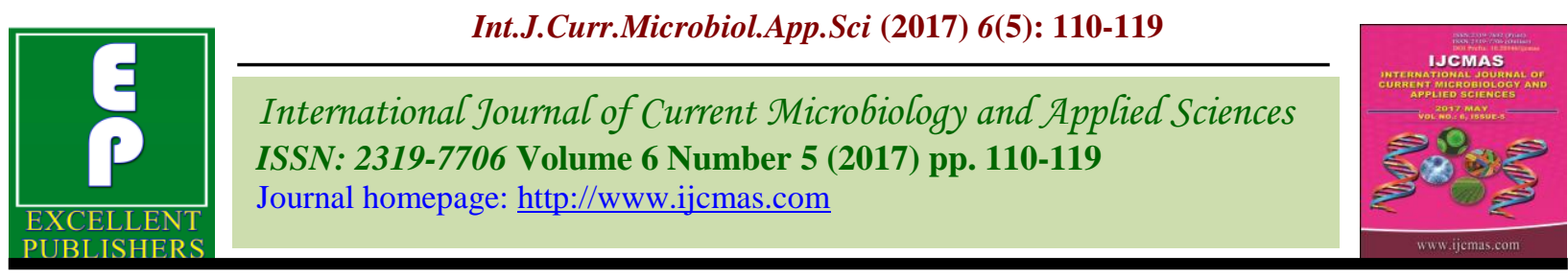

Original Research Article

https://doi.org/10.20546/ijcmas.2017.605.014

\title{
Cloning and Identification of an $A C O$ Gene Involved in Ethylene Production Pathway from Ginkgo biloba
}

\author{
Jiaping Yan, Weiwei Zhang*, Feng Xu and Yongling Liao \\ College of Horticulture and Gardening, Yangtze University, Jingzhou, 434025, China \\ *Corresponding author
}

\begin{tabular}{|c|c|}
\hline & A B S T R A C T \\
\hline Keywords & \multirow{8}{*}{$\begin{array}{l}\text { 1-Aminocyclopropane-1-carboxylic acid (ACC) oxidase (ACO), is one of the core } \\
\text { enzymesin the ethylene biosynthesis pathway, which catalyzes the final step in ethylene } \\
\text { biosynthesis converting ACC to ethylene, cyanide, } \mathrm{CO}_{2} \text {, dehydroascorbate and water with } \\
\text { inputs of } \mathrm{Fe}(\mathrm{II}) \text {, ascorbic, bicarbonate and oxygen.In the present study, a } 1288 \mathrm{bp} \mathrm{cDNA} \\
\text { sequence fragment of the } A C O \text { gene (designated as } G b A C O \text { ) was isolated from ginkgo } \\
\text { (Ginkgo biloba) leaves by using reverse transcriptase-polymerase chain reaction (RT-PCR) } \\
\text { with two PCR primers designed according to the ginkgo transcriptome data. This cDNA } \\
\text { sequence contained a 990bp open reading frame (ORF), which encoding a } 329 \text {-amino-acid } \\
\text { protein. The molecular weight and isoelectric point are } 37.2 \mathrm{kDa} \text { and } 5.20 \text {, respectively. } \\
\text { The encoding amino-acid sequence showed high similarity to other plant ACO proteins. } \\
\text { The secondary structure prediction indicated that GbACO protein comprised ofa-helix } \\
\text { ( } 40.43 \% \text { ), } \beta \text {-turn (10.33\%), extended strand ( } 13.07 \% \text { ), and random coil ( } 36.17 \% \text { ). } \\
\text { Phylogenetic tree analysis revealed that GbACO clustered with the ACO in the } \\
\text { Gymnospermae clade.The isolation and characterization analysis of GbACO is helpful to } \\
\text { understand the biosynthesis of ethylene in } G \text {. biloba at the molecular level and also } \\
\text { provides some theoretical support for improving the ethvlene production. }\end{array}$} \\
\hline & \\
\hline $\begin{array}{l}\text { Cyclo propane-1- } \\
\text { carboxylic acid }\end{array}$ & \\
\hline & \\
\hline Gene cloning, & \\
\hline & \\
\hline Article Info & \\
\hline $\begin{array}{l}\text { Accepted: } \\
\text { 04 April } 2017 \\
\text { Available Online: } \\
10 \text { May } 2017\end{array}$ & \\
\hline
\end{tabular}

\section{Introduction}

Ginkgo biloba is a living fossil from the Cenozoic Quaternary glacial period. Itis a precious tree species in China and has been extensively studied given its important medical and ornamental value. It has a long juvenile phase and spends 15 to 20 years in the flowering transition, and ethylene has played a very important role in its life cycle.As we all know, ethylene, one of the most important plant hormones, involves in the various aspects of plant growth and development, such as seed dormancy and germination, cell elongation, root growth and nodulation, shoot and leaf formation, flower and fruit development, sexual development, plant defense mechanism, and so on (Abeles et al., 1992;Wang et al., 2002; Chaves and Mello-Farias, 2006; Argueso et al., 2007; Lin et al., 2009). Although ethylene is plays an essential role for proper plant growth, development, and survival, it may also be deleterious to plants in some instances. Ethylene is biologically active in trace amounts, and the effects of this hormone are commercially important (Yang and Hoffman, 1984). Lieberman and Mapson (1964), Murr and Yang (1975), Adams and Yang (1979) made major contributions to our 
understanding of ethylene biosynthesis. A simplified overview of the pathway is presented in figure 1. The number of sequenced lower plant genomes has increased extensively in the past few years, providing opportunities to trace the evolutionary origins of biosynthetic and signaling pathways of plant hormones (Ross and Reid, 2010; Ju et al., 2015). The ethylene biosynthetic pathway, which includes three key enzymes reactions, has been well-documented in higher plants. First, the biosynthesis of ethylene begins with the conversion of the amino acid methionine to S-adenosyl-methionine (SAM) via enzyme SAM synthase (Yang and Hoffman, 1984; Zarembinski and Theologis, 1994). Second, the rate-limiting step catalyzed by 1aminocyclopropane-1-carboxylic acid (ACC) synthase (ACS) involves the cyclization of SAM to ACC, and 5'-methylthioadenosine (MTA) is produced by the ACS to utilize the synthesis of new methionine by the activated methyl cycle. Finally, ACC oxidase (ACO) catalyzes the oxygen-dependent conversion of ACC to ethylene (Yang and Hoffman, 1984; Liuet al., 1999; Choudhury et al., 2008).

Increased ethylene levels in plants when exposed to various types of stress including chilling, heat, nutrient deprivation, anaerobic life, wounding, and pathogen infection with increased damage to plant growth and health has been reported (Stearms and Glick, 2003). There is considerable commercial interest in modifying the amount of ethylene produced under ripening, senescent or stress conditions and thereby creating plants with more robust and/or desirable traits (Handa et al., 2010). The level of ethylene in plants is determined by the activity of two enzymes that catalyze the two final reaction steps in the biosynthetic pathway: ACS and ACO gene. Both ACS and ACO are encoded by multigene families that are differentially regulated by diverse developmental and environmental inputs (Barry et al., 1996; Chen and McManus,
2006), the majority of the regulatory mechanisms of ethylene biosynthesis at the level of ACC production by ACC. However, there are additional regulatory mechanisms. Under conditions of high ethylene production, the pathway can also be regulated at the level of conversion of ACC into ethylene by ACO (Vanderstraeten and Van Der Straeten, 2017). ACO enzyme belongs to the family of oxidoreductases which utilize Fe (II) as a cofactor and 2-oxoglutarases (2OG) as cosubstrate, although ACO uses ascorbate as a co-substrate (Prescott, 1993; Ryle and Hanusinger, 2002; Zhang et al., 2004; Mirica and Klinman, 2008). ACO needs bicarbonate as an activator and catalyzes the oxidation of ACC to give ethylene, $\mathrm{CO}_{2}$, and $\mathrm{HCN}$. It has been revealed that $\mathrm{ACO}$ forms a complex with $\mathrm{Fe}$ (II) and its active site contains a single $\mathrm{Fe}$ (II) matched with three residues (Zhang et al., 2004; Jafari et al., 2013). In its sequence, two distinct domains can be distinguished: an N-terminal located highly conservative non-heme dioxygenase DIOX_N region and a C-terminal located 2OGFe II_Oxy region.

The practical implication of ethylene biosynthesis regulation for plant improvement has supported the continuous basic research on dissecting the structure of genes encoding ethylene biosynthetic enzymes, their differential expression patterns, and mechanisms underlying their transcriptional activity (Izabela et al., 2013). ACO has been cloned and characterized in many plant species such as peach, potato, strawberry, white clover, plum, Arabidopsis, rice, apple, pine, pear (Ruperti et al., 2001; Nie et al., 2002; Trainotti et al., 2005; Chen and McManus, 2006; Fernandez-Otero et al., 2006; Babula et al., 2006; Quyang et al., 2007; Binnie and McManus, 2009; Yuan et al., 2010; Qi et al., 2015). Here, we identified the $G b A C O$ gene originated from the $G$. bilobaleaves that promoted the growth of 
ginkgo, and analyzed the sequence structure and function. We constructed a phylogenetic tree for the comparison of GbACO with other members of the plant dioxygenase family through the neighbor-joining method. These results revealed that GbACO is a key enzyme gene in the ethylene biosynthesis pathway, and also laid a foundation for the analysis of gene regulatory network of ethylene synthesis in the G. biloba.

\section{Materials and Methods}

\section{Plant materials}

The 15-years-old G. biloba plants were grown in the Ginkgo Garden of Yangtze University (Jingzhou, China). Ginkgo leaves were immediately frozen in liquid nitrogen at the time of collection and then stored at $-80^{\circ} \mathrm{Cfor}$ further use. Both primers synthesis and DNA sequencing were performed by Shanghai Sangon Biotechnology Company, China.

\section{Cloning of $A C O$ homologous from $G$. biloba}

Total RNA was extracted from frozen ginkgo leaves using the TaKaRa MiniBEST Plant RNA Extraction kit (Dalian, China). First strand cDNA was synthesized using TaKaRa PrimeScript $^{\mathrm{TM}} 1$ st Strand cDNA Synthesis Kit according to the manufacturer's instruction. A set of primers GbACO-up (5'CTCATAACAATAATAGAGATTACTTTC TCA-3') and GbACO-dn (5'TTAAGATTTGAACTAAAGTAAGATTGT -3') were designed based on the previous ginkgo transcriptome data (CL1905Contig1). Each $25 \mu \mathrm{l}$ reaction volume contained $0.5 \mu \mathrm{g}$

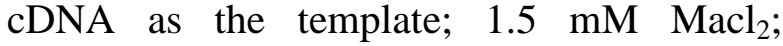
$10 \times$ Taq buffer; $0.4 \mu \mathrm{M}$ of each primer; 0.25 $\mathrm{mM}$ dNTPs; and $0.25 \mathrm{U}$ Taq polymerase. The reactions were initiated at $94^{\circ} \mathrm{C}(4 \mathrm{~min})$, followed by 35 cycles of denaturing at $94^{\circ} \mathrm{C}$ (1 min), annealing at $53.5^{\circ} \mathrm{C}(50 \mathrm{~s})$, and elongation at $72^{\circ} \mathrm{C}(1 \mathrm{~min})$, and ended with incubation at $72^{\circ} \mathrm{C}$ for $10 \mathrm{~min}$. The PCR products were separated on a $1 \%(\mathrm{w} / \mathrm{v})$ agarose gel, then the target DNA (with the expected size) was excised from the gel and purified using TaKaRa Agarose Gel DNA purification Kit Ver.4.0. The purified products were ligated into the pMD19-T plasmid vector (TaKaRa), and then cloned into the Escherichia coli DH5 $\alpha$ followed by sequencing.

\section{Bioinformatics and molecular evolution analyses}

The obtained nucleotide sequence from sequencing was analyzed and translated using Vector NTI 11.5 program, and the predictions for $\mathrm{pI}$ and molecular mass were using the $\mathrm{pI} / \mathrm{MW}$ tool at Expasy (http://web.expasy.org/compute_pi/).

Secondary structure was predicted by SOPMA (https://npsa-prabi.ibcp.fr/cgibin/npsa_automat.pl?page=/NPSA/npsa_sop ma.html). The deduced protein sequence was searched for homologous proteins using the BLAST-Basic Local Alignment Search Tool (http://www.ncbi.nlm.nih.gov/BLAST/).

Sequences with the highest similarities were downloaded and multiple alignments were performed using Vector NTI 11.5 program, and the phylogenetic tree was constructed using Clustal X2 and MEGA 6.0 software.

\section{Results and Discussion}

\section{Cloning and characterization of $G b A C O$}

In order to characterize the full-length GbACOc DNA, total RNA was isolated from ginkgo leaves. cDNA was reverse transcribed from RNA, and then, the corresponding GbACOcDNA amplification was performed using the primers (GbACO-up and GbACO$\mathrm{dn})$, designed based on previous transcriptome data (CL1905Contig1). A single PCR fragment of the expected size was 
obtained, purified, ligated into the pMD19-T vector, and then cloned into the $E$. coli $\mathrm{DH} 5 \alpha$, finally sequenced. Sequencing results showed that the full-length cDNA of GbACO was 1288 bp and contained a 990bp ORF, which encoding a 329-amino-acid protein (Figure 2). The molecular weight and isoelectric point of the deduced polypeptide were $37.2 \mathrm{kDa}$ and 5.20 , respectively.

\section{Characterization of the deduced GbACO protein}

The comparison of the amino acid sequence of GbACO with other plant ACO proteins showed high sequence similarity. The GbACO protein showed 64\%, 62\%, 61\%, $60 \%, 60 \%, 59 \%, 60 \%, 63 \%$, and $61 \%$ similarity to the counterparts of Picea sitchensis (ABF20552.1), Pseudotsuga menziesii (ABF20554.1), Elaeis guineensis (XP_010905322.1), Phoenix dactylifera (XP_008805862.1), Nelumbo nucifera (XP_010269058.1), Ficus carica (AKJ87578.1), Cucumis melo (XP_008444197.1), Pinus pinaster (CBL95267.1), and Ricinus communis (XP_002520313.1), respectively. Multiple sequence alignments showed that the peptide is a member of ACO proteins, with DIOX_N (non-haem dioxygenase in morphine synthesis N-terminal), and 2OG-Fe II_Oxy (2oxoglutarate (2OG) and Fe (II)-dependent oxygenase) domains (Figure 3). As we all know, ACO is a member of Fe (II)-dependent family of oxidases, which requires ascorbate as a co-substrate and Fe (II) as a cofactor for its enzymatic activity (McGarvey and Christoffersen, 1992). All motifs for binding of the cofactor (His-Xaa-Asp-Xaa-His) and the co-substrate (Arg-Xaa-Ser) that are highly conserved among all members of $\mathrm{Fe}$ (II) ascorbate family of dioxygenases were also well conserved in GbACO and other plant ACO proteins (Figure. 3). The Fe2OG dioxygenases catalyze a variety of twoelectron oxidations, including hydroxylations, desaturations, and oxidative ring closures (Costas et al., 2004). Although the sequence homology among these enzymes is not high, all their active sites contain a single ferrous ion bound in a tridentate ligand arrangement referred to as a "2-His-1-carboxylate facial triad" (Mirica and Klinman, 2008). Secondary structure analysis of $\mathrm{GbACO}$ protein by SOMPA revealed that GbACO consists of $\alpha$ helix (40.43\%), $\beta$-turn $(10.33 \%)$, extended strand $(13.07 \%)$, and random coil $(36.17 \%)$.

Figure. 1 Ethylene biosynthesis pathway. The enzymes catalyzing each step are shown above the arrows. The methionine is converted to $\mathrm{S}$-adenosyl-methionine (SAM) via enzyme SAM synthase (SAMS). SAM is subsequently converted to ACC by ACC synthase (ACS). MTA finally, ACC is converted to ethylene by ACC oxidase (ACO). (Yang and Hoffman, 1984;

Vanderstraeten and Van Der Straeten, 2017)

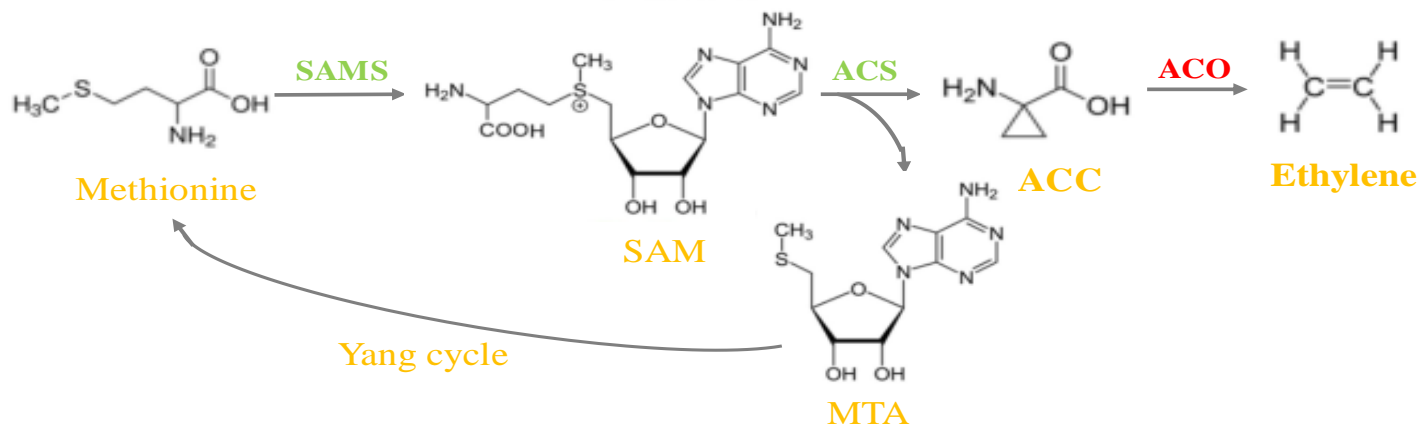


Figure. 2 cDNA sequenceof $G b A C O$ and the translated amino acid sequence. The red box is the start codon, and the asterisk represents the termination codon

\begin{tabular}{|c|c|}
\hline 76 & $\begin{array}{l}\text { ctcataacaataatagagattacttctcattcttctttgaatacttcatt getcttgtgagtctttcttagt } \\
\text { aatacaaataATGGCTATTCCGTGATTGACATGGCGATCTCACTGGAGAAAGTAGAGCCATCACCATGGCTCA }\end{array}$ \\
\hline & 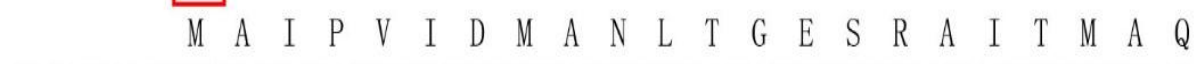 \\
\hline & ATAGAAAATGGGTGCCAGGAATGGGGCTTCATGCAGCTTTTGAACCACGGCATACCTACTACTCTATTGGACAG \\
\hline & $\begin{array}{lllllllllllllllllllllllll}I & E & N & G & C & Q & E & \mathbb{W} & G & F & M & Q & L & L & N & H & G & I & P & T & T & L & L & D & S\end{array}$ \\
\hline & CGTTAAGAGAGTATGCTCTGAGAATTATAAGCTTATCAGAGAGAAAGAGTTCAGTGAGTCATTCCCAGTGAAAAT \\
\hline & 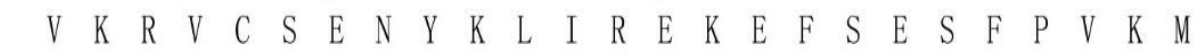 \\
\hline & GCTTAATAATGCCCTCGTGGAAGCTGCGAATAATGGCTCTACAGAGCCAAAAAAGCTAGACAATGTGGACTGGGA \\
\hline & $\begin{array}{lllllllllllllllllllllllll}L & N & N & A & L & V & E & A & A & N & N & G & S & T & E & P & K & K & L & D & N & V & D & W & E\end{array}$ \\
\hline & AGATGTGTTTGTGATCAACTACATGCAAGAGTCCTACGTGTGGCCTTCGGAGCCCAGCGATTTCAGGGAAACAAT \\
\hline & 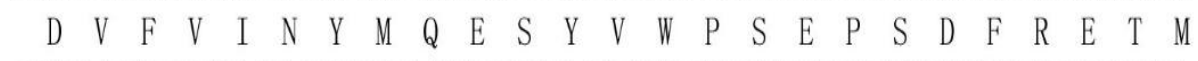 \\
\hline & GGAGAAATTGGGAAAGGAGGTGTACAAATTGGCAGAGAAATTGCTAGAGCTGCTGAGTGAGAATTTGGGTTTGGA \\
\hline & 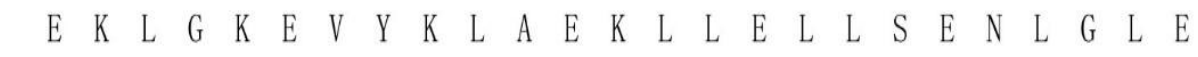 \\
\hline & GAAAGACTATATTAAGAAAGCATTTGCAGGGGGAAGTGGAACTGATCACAAGCCCTTTTTCGGTACTAAAGTTAG \\
\hline & 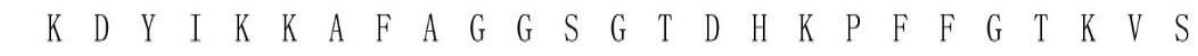 \\
\hline & CCATTATCCACCGTGCCCTAGACCAGACCTTATCACTGGTCTACGTGCGCACACTGATGCTGGCGGACTGATTCT \\
\hline & 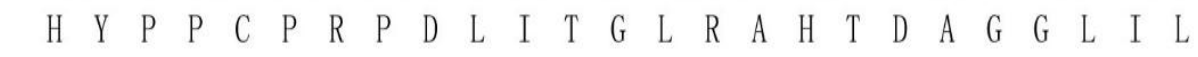 \\
\hline & GCTGTATCAAGATGATGAAGTAGCCGGTCTTCAGGTCCTCAAGGATGGTCATTGGTTCGATGTACAACCGATGCC \\
\hline & 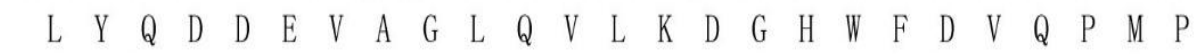 \\
\hline & CAACGCAATTGTTATTGACATTGGTGATCAGTTGGAGGCGATTAGCAATGGTAGATATAAGAGCGCATGGCATCG \\
\hline & $\begin{array}{lllllllllllllllllllllllll}N & A & I & V & I & D & I & G & D & Q & L & E & A & I & S & N & G & R & Y & K & S & A & W & H & R\end{array}$ \\
\hline & TGTGCTGCCTAATGAAAATGGCACTCGAAGGTCAGTGGCATCGTTTTACAACCCATCATACGATGCAGTGGTGTA \\
\hline & 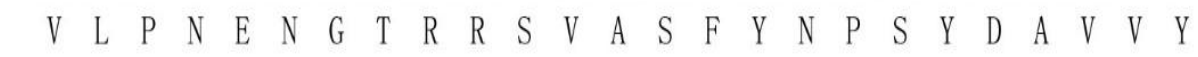 \\
\hline & TCCTGCTCCACAACTAATGACTCCAAAGCTATCAGCGGATAAGGAGGATGATCACAATGGTGCTGAGTCATCGCT \\
\hline & $\begin{array}{lllllllllllllllllllllllll}P & A & P & Q & L & M & T & P & K & L & S & A & D & K & E & D & D & H & N & G & A & E & S & S & L\end{array}$ \\
\hline & TTATCCAAAGTTTCTGTTTGCTGATTATATGAGTGTTTATGCTCATCAGAAATATCTTCCAAAAGAGCCGCGATT \\
\hline & 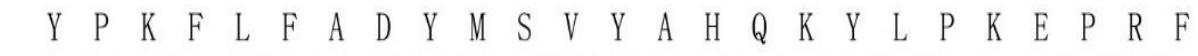 \\
\hline & CCAAGCTATGAGAGCCTTGTATTAGCCTACAGTCATAGGTTATTCATAGATAATAGTAGTTTATATTTCATGGTA \\
\hline & 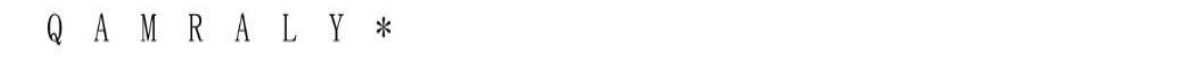 \\
\hline & tattagcctacagtcataggttattcatagataatagtagtttatatttcatggtagccttgtattagc \\
\hline & tcataggttattcatagataatagtgtttatatttcatgttattgggttcatcaaacaatcttacttta \\
\hline & \\
\hline
\end{tabular}


Figure.3 Alignment of the deduced GbACO amino acid sequence with other ACO proteins., The species names are shown as following: GbACO, G. biloba; EgACO, E. guineensis; PdACO, $P$. dactylifera; CmACO, C. melo; RcACO, R. communis; NnACO, N. nucifera; FaACO, F. carica; PmACO, P. menziesii; PpACO, P. pinaster; PsACO, P. sitchensis. Green and red boxes are represented DIOX_N and 2OG-Fe II-Oxy domains, respectively. All conserved amino acids important for ACO activity are labeled in red color and are conserved in all members of theFe (II) ascorbate family of dioxygenases

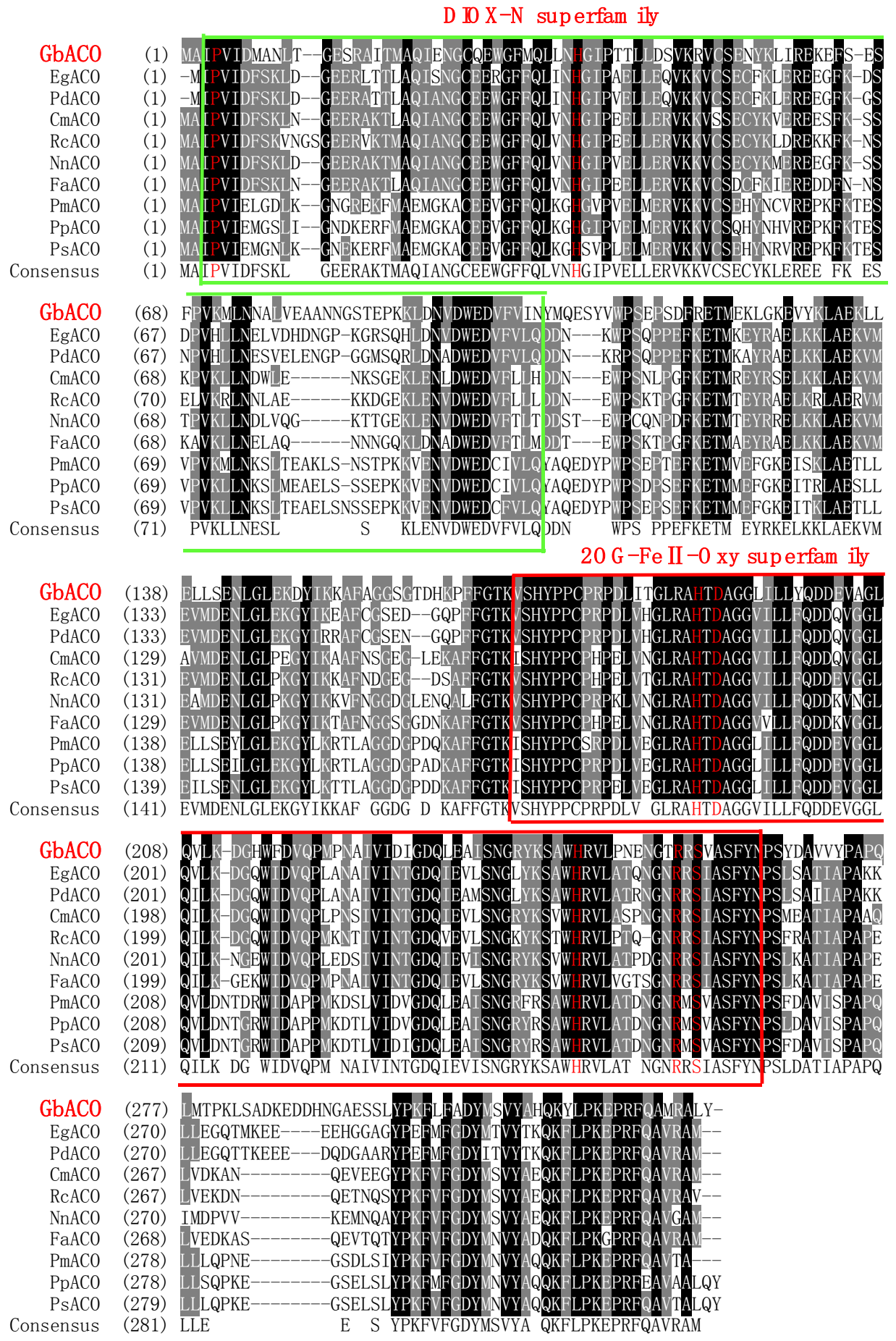


Figure.4 Phylogenetic relationships between GbACO and other plant ACO proteins., The phylogenetic tree was constructed by use of Clustal X2 and MEGA 6.0 software with the neighbor-joining method based on the amino acid sequences multiple alignments. The species names are listed as following: PtACO, Populus tremula $x$ Populus tremuloides; PtrACO, $P$. trichocarpa; RcACO, R. communis; JcACO, Jatropha curcas; PxACO, Pyrus x bretschneideri; FvACO, Fragaria vesca subsp. Vesca; PmuACO, Prunus mume; GhACO, Gossypium hirsutum; GrACO, G. raimondii; GmACO, Glycine max; VrACO, Vigna radiata var. radiate; AiACO, Arachis ipaensis; MtACO, Medicago truncatula; CsACO, C. sativus; CmACO, C. melo; MnACO, Morus notabilis; FaACO, F. carica; NsACO, Nicotiana sylvestris; CanACO, Capsicum annuum; StACO, Solanum tuberosum; BnACO, Brassica napus; BrACO, B. rapa; EgACO, E. guineensis; PdACO, P. dactylifera; ZmACO, Zea mays; SbACO, Sorghum bicolor; GbACO, G. biloba; PsACO, P. sitchensis; PpACO, P. pinaster; PmACO, P. menziesii

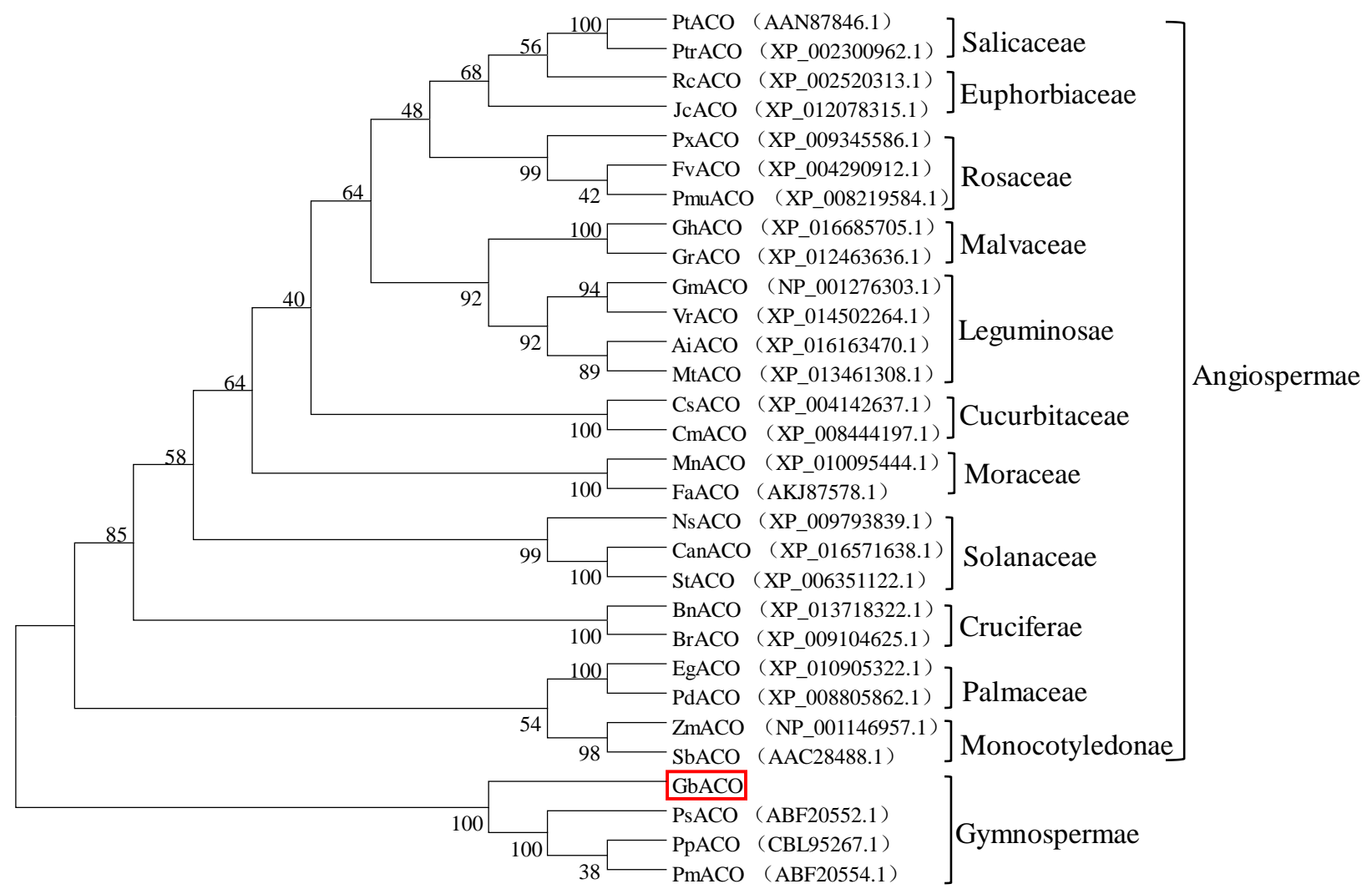

\section{Molecular evolution analysis}

In order to better understanding the molecular evolution relationship of GbACO protein, a phylogenetic tree was constructed from an alignment of the deduced amino acid sequence from the ginkgo ACO with other plant ACO proteins via the neighbor-joining method with the Cluxtal X2 and MEGA 6.0 software. As shown in figure $4, \mathrm{GbACO}$ was classified into the Gymnospermae branch; all the species showing such a high degree of homology belonged to the Gymnospermae. Thus, GbACO protein has its closest relationship to Gymnosperms, which form a separate cluster on the tree, while another cluster consists of the ACO proteins from Angiosperms, including eleven families (Salicaceae, Euphorbiaceae, Rosaceae, Malvaceae, Leguminosae, Cucurbitaceae, 
Moraceae, Solanaceae, Cruciferae, Palmaceae and Monocotyledonae). Taken together, phylogenetic analysis suggests that GbACO has a common evolutionary original with other plant ACO proteins based on the conserved sequence and sequence characteristics.

\section{Conclusion and perspectives}

ACO catalyzes the final step in the biosynthesis of the plant signaling molecule ethylene that is involved in the regulation of array of biological processes in plants, including seed dormancy and germination, root growth, shoot and leaf formation, flower and fruit development, different organs senescence, plant defense mechanisms, and a number of interactions with other plant hormones. Here, an ACO homologue, GbACO was successfully isolated and characterized for the first time from ginkgo. Multiple sequence alignments indicated that GbACO had a high similarity to other plant ACO proteins. Phylogenetic tree analysis showed that GbACO keeps a strong conservation during the molecular evolution. All of these results not only contribute to understand the ethylene biosynthesis in Gymnosperms but also promote researches of the role of ethylene in the regulation of ginkgo growth and development.

Nowadays, the number of sequenced plant genomes has increased extensively in the past few years, providing opportunities to probe the evolutionary origins of biosynthetic and signaling pathways of plant hormones. There is a commercial interest in increasing the final products through increasing the expression level of key genes in the biosynthesis pathway. Even though ACO genes have been isolated from a lot of species, the biochemical properties, in vivo stability, and/or physiological functions of the gene products are in many cases not fully understood. Thus, further study is needed to verify the role played by different $A C O$ genes in the regulation of ethylene production. A better understanding of both structure and function of genes involved in the ethylene biosynthesis, and also the mechanisms responsible for their activity during different developmental processes of plants. All this has a profound practical importance as it develops the tools for ginkgo improvement.

\section{Acknowledgment}

This study was supported by the National Natural Science Foundation of China (31500546), the Doctor Foundation of Yangtze University (801190010127).

\section{References}

Abeles, F.B.M., Morgan, P.W., and Saltveit, M.E. 1992. Ethylene in Plant Biology. San Diego, CA: Academic Press.

Adams, D.O., and Yang, S.F. 1979. Ethylene biosynthesis: identification of 1aminocyclopropane-1-carboxylic acid as an intermediate in the conversion of methionine to ethylene. Proceedings of the National Academy of Sciences, 76(1): 170-174.

Argueso, C.T., Hansen, M., and Kieber, J.J. 2007. Regulation of ethylene biosynthesis. J. Plant Growth Regulation, 26(2): 92-105.

Babula, D., Misztal, L. H., Jakubowicz, M., Kaczmarek, M., Nowak, W., and Sadowski, J. 2006. Genes involved in biosynthesis and signalisation of ethylene in Brassica oleracea and Arabidopsis thaliana: identification and genome comparative mapping of specific gene homologues. Theoretical and Appl. Genetics, 112(3): 410-420.

Barry, C.S., Blume, B., Bouzayen, M., Cooper, W., Hamilton, A.J., and Grierson, D. 1996. Differential expression of the 1aminocyclopropane-1-carboxylateoxidase gene family of tomato. The Plant J., 9(4): 
525-535.

Binnie, J.E., and McManus, M.T. 2009. Characterization of the 1aminocyclopropane-1-carboxylic acid (ACC) oxidase multigene family of Malus domestica Borkh. Phytochem., 70(3): 348-360.

Chaves, A.L.S., and Mello-Farias, P.C.D. 2006. Ethylene and fruit ripening: from illumination gas to the control of gene expression, more than a century of discoveries. Genetics and Mol. Biol., 29(3): 508-515.

Chen, B.C.M., and McManus, M.T. 2006. Expression of 1-aminocyclopropane-1carboxylate (ACC) oxidase genes during the development of vegetative tissues in white clover (Trifolium repens L.) is regulated by ontological cues. Plant Mol. Biol., 60(3): 451-467.

Choudhury, S.R., Roy, S., Saha, P.P., Singh, S.K., and Sengupta, D.N. 2008. Characterization of differential ripening pattern in association with ethylene biosynthesis in the fruits of five naturally occurring banana cultivars and detection of a GCC-box-specific DNA-binding protein. Plant Cell Reports, 27(7): 1235.

Costas, M., Mehn, M.P., Jensen, M.P., and Que, L. 2004. Dioxygen activation at mononuclear nonheme iron active sites: enzymes, models, and intermediates. Chem. Rev., 104(2): 939-986.

Fernández-Otero, C., Matilla, A.J., Rasori, A., Ramina, A., and Bonghi, C. 2006. Regulation of ethylene biosynthesis in reproductive organs of damson plum (Prunus domestica L. subsp. Syriaca). Plant Sci., 171(1): 74-83.

Handa, A.K., Srivastava, A., Deng, Z., Gaffe, J., Arora, A., Tiznado-Hernandez, M.E., Goyal, R.K., Malladi, A., Negi, P.S., and Mattoo, A. K. 2010. Biotechnological interventions to improve plant developmental traits. Transgenic Crop Plants, 199-248.

Jafari, Z., Haddad, R., Hosseini, R., and Garoosi, G. 2013. Cloning, identification and expression analysis of ACC oxidase gene involved in ethylene production pathway. Mol. Biology Reports, 40(2): 1341-1350.

Ju, C., Van de Poel, B., Cooper, E.D., Thierer, J.H., Gibbons, T.R., Delwiche, C.F., and Chang, C. 2015. Conservation of ethylene as a plant hormone over 450 million years of evolution. Nature Plants, 1, 14004.

Lieberman, M., and Mapson, L.W. 1964. Genesis andbiogenesis of ethylene. Nature, 204: 343-345.

Lin, Z., Zhong, S., and Grierson, D. 2009. Recent advances in ethylene research. $J$. Experimental Botany, 60(12): 3311-3336.

Liu, X., Shiomi, S., Nakatsuka, A., Kubo, Y., Nakamura, R., and Inaba, A. 1999. Characterization of ethylene biosynthesis associated with ripening in banana fruit. Plant Physiol., 121(4): 1257-1265.

Mirica, L.M., and Klinman, J.P. 2008. The nature of $\mathrm{O} 2$ activation by the ethyleneforming enzyme 1-aminocyclopropane-1carboxylic acid oxidase. Proceedings of the National Academy of Sciences, 105(6): 1814-1819.

Murr, D.P., and Yang, S.F. 1975. Conversion of 5'-methylthioadenosine to methionine by apple tissue. Phytochem., 14(5-6): 12911292.

Nie, X., Singh, R.P., and Tai, G.C. 2002. Molecular characterization and expression analysis of 1aminocyclopropane-1-carboxylate

oxidase homologs from potato under abiotic and biotic stresses. Genome, 45(5): 905-913.

Ouyang, S., Zhu, W., Hamilton, J., Lin, H., Campbell, M., Childs, K., ThibaudNissen, F., Malek, R. L., Lee, Y., Zheng, L., Orvis, J., Haas, B., Wortman, J., and Buell, C.R. 2007. The TIGR rice genome annotation resource: improvements and new features. Nucleic Acids Res., 35(suppl 1): D883-D887.

Prescott, A.G. 1993. A dilemma of dioxygenases (or where biochemistry and molecular biology fail to meet). $J$. Experimental Botany, 44(5): 849-861.

Qi, J., Dong, Z., and Zhang, Y.X. 2015. 
Complementary DNA cloning of the pear 1-aminocyclopropane-1-carboxylic acid oxidase gene and agrobacterium-mediated anti-sense genetic transformation. Mol. Med. Reports, 12(6): 8268-8274.

Ross, J.J., and Reid, J.B. 2010. Evolution of growth-promoting plant hormones. Functional Plant Biol., 37(9): 795-805.

Ruduś, I., Sasiak, M., and Kępczyński, J. 2013. Regulation of ethylene biosynthesis at the level of 1-aminocyclopropane-1carboxylate oxidase (ACO) gene. Acta Physiologiae Plantarum, 35(2): 295-307.

Ruperti, B., Bonghi, C., Rasori, A., Ramina, A., and Tonutti, P. 2001. Characterization and expression of two members of the Peach 1-aminocyclopropane-1carboxylate oxidase gene family. Physiologia Plantarum, 111(3): 336-344.

Ryle, M. J., and Hausinger, R. P. 2002. Nonheme iron oxygenases. Curr. Opinion in Chem. Biol., 6(2): 193-201.

Stearns, J.C., and Glick, B.R. 2003. Transgenic plants with altered ethylene biosynthesis or perception. Biotechnol. Adv., 21(3): 193-210.

Trainotti, L., Pavanello, A., and Casadoro, G. 2005. Different ethylene receptors show an increased expression during the ripening of strawberries: does such an increment imply a role for ethylene in the ripening of these non-climacteric fruits? J. Experimental Botany, 56(418): 2037-
2046.

Vanderstraeten, L., and Van Der Straeten, D. 2017. Accumulation and transport of 1aminocyclopropane-1-carboxylic acid (ACC) in plants: current status, considerations for future research and agronomic applications. Frontiers in Plant Sci., 8: 38.

Wang, K.L.C., Li, H., and Ecker, J.R. 2002. Ethylene biosynthesis and signaling networks. The Plant Cell, 14(suppl 1): S131-S151.

Yang, S.F., and Hoffman, N.E. 1984. Ethylene biosynthesis and its regulation in higher plants. Annual Rev. Plant Physiol., 35(1): 155-189.

Yuan, S., Wang, Y., and Dean, J.F.D. 2010. ACC oxidase genes expressed in the wood-forming tissues of loblolly pine (Pinus taeda L.) include a pair of nearly identical paralogs (NIPs). Gene, 453(1): 24-36.

Zarembinski, T.I., and Theologis, A. 1994. Ethylene biosynthesis and action: a case of conservation. Plant Mol. Biol., 26(5): 1579-1597.

Zhang, Z., Ren, J.S., Clifton, I.J., and Schofield, C.J. 2004. Crystal structure and mechanistic implications of 1aminocyclopropane-1-carboxylic acid oxidase - the ethylene-forming enzyme. Chem. Biol., 11(10): 1383-1394.

\section{How to cite this article:}

Jiaping Yan, Weiwei Zhang, Feng Xu and Yongling Liao. 2017. Cloning and Identification of an ACO Gene Involved in Ethylene Production Pathway from Ginkgo biloba. Int.J.Curr.Microbiol.App.Sci. 6(5): 110-119. doi: http://dx.doi.org/10.20546/ijcmas.2017.605.014 